\title{
Tinea corporis in a mixed martial arts fighter
}

\author{
Claude Bachmeyer MD, Geneviève Buot MD
}

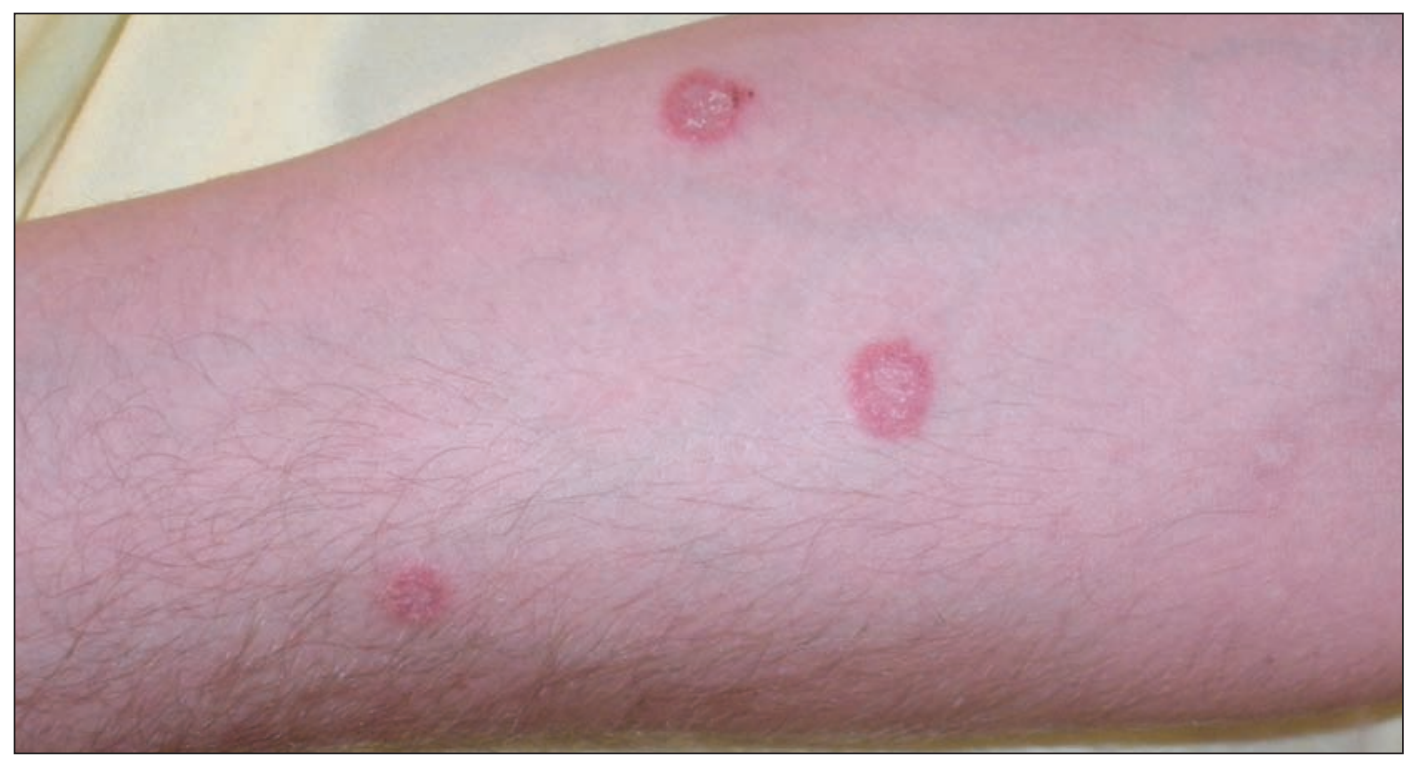

Competing interests: None declared.

This article has been peer reviewed.

\section{Affiliations: From the Department of Internal Medicine (Bachmeyer), Tenon Hospital (Assistance Publique-Hôpitaux de Paris); and the Laboratory of Parasitology-Mycology (Buot), Saint-Antoine Hospital (Assistance Publique-Hôpitaux de Paris) Paris, France \\ Correspondence to: Claude Bachmeyer, claude .bachmeyer@tnn.aphp.fr}

CMAJ 2013. DOI:10.1503 /cmaj.120813

Figure 1: Erythematous and scaly lesions with healing centres and active peripheries on the forearm of a 30-year-old mixed martial arts fighter.

A 30-year-old mixed martial arts fighter whose training partners had recently received diagnoses of tinea corporis presented with a 4 -week history of pruritic cutaneous lesions. He had no relevant medical history. On examination, we saw about 12 welldefined, erythematous and scaly lesions on his arms and neck (Figure 1). The remainder of the physical examination was unremarkable. Microscopic examination of skin scrapings in potassium hydroxide showed dermatophytic hyphae. Mycologic cultures grew Trichophyton tonsurans. Following local treatment with bifonazole and oral griseofulvin for 6 weeks, the lesions resolved completely.

Tinea corporis is a common infection among athletes participating in combat sports. It is most frequently related to $T$. tonsurans, an anthropophilic dermatophyte spread by close skin-toskin contact. Whereas the prevalence of this infection among mixed martial arts fighters is not known, tinea corporis has been described in $11 \%-14 \%$ of judo fighters and occurs even more frequently among wrestlers, from whom it gets the name tinea corporis gladiatorum. ${ }^{1,2}$ The diagnosis of tinea corporis is primarily clinical and can be confirmed by microscopic examination and mycologic cultures. Treatment is topical (e.g., azoles, allylamines, ciclopirox olamine) for localized lesions and systemic (e.g., azoles, terbinafine) for more diffuse lesions and hair infection. ${ }^{1,2}$ According to the 2010 position statement of the National Athletic Trainers' Association, active fungal lesions must be treated topically for a minimum of 72 hours or adequately covered before competition. Failure to follow these recommendations is considered grounds for disqualification. ${ }^{3}$

\section{References}

1. Bassiri-Jahromi S, Sadeghi G, Paskiaee FA. Evaluation of the association of superficial dermatophytosis and athletic activities with special reference to its prevention and control. Int J Dermatol 2010;49:1159-64

2. Hirose N, Suganami M, Ogawa YS, et al. Screening examination and treatment of Trichophyton tonsurans infection in judo athletes affiliated with the University Judo Federation of Tokyo. Mycoses 2011;54:e35-8

3. Zinder SM, Basler RSW, Foley J, et al. National Athletic Trainers Association position statement: skin diseases. J Athl Train 2010; 45:411-28. 